\section{A program for simultaneous stimulation and data handling in neurophysiological} research

\author{
J. MICHAUX and J. BERGMANS* \\ Electronics Research Laboratory and Department of Neurology \\ University of Louvain, Belgium
}

The possibilities for on-line automatic control of experiments in a laboratory environment have greatly increased since the development of small general-purpose computers. In the neurophysiological field, the experimental tasks may be subdivided into four steps: (1) analysis routines have to be applied to (2) data recorded in response to (3) stimuli applied to the preparation according to (4) an experimental program devised in the light of previous results of similar experiments and modified as prescribed by the actual results. Most currently, the use of the computer has been limited to the two first tasks, the computer recording the data and submitting them on-line to some analysis programs (e.g., transient averaging routines, etc.). It may be envisaged to take over also the execution of the latter tasks; and as a first step, this communication is concerned specifically with a program which controls entirely the sequences of stimuli given to a preparation during a whole experiment, and at the same time executes analysis routines on the events occurring in response to the stimuli.

\section{THE PROGR AMMED PULSE GENERATOR ${ }^{1}$}

Method

A program, called "programmed pulse generator" (PPG) has been written on a PDP-8 computer (Digital Equipment Corp.). It allows one to issue, through two digital-to-analog converters connected to the computer, any sequence of pulses needed for experimental purposes, and to interlace specified routines during intervals between pulses. In the present version of PPG, the only controlled variable in the pulse series is the interval between the pulses. The pulses issued by the computer are either used as such for synchronization purposes or else they are shaped in pulses suitable for stimulation of nervous structures by triggering a conventional stimulator (Grass Type S8). In such a way, the amplitude and the duration of the stimulating pulses can be adapted manually for each experiment, but there is as yet no possibility for automatic control of those parameters by the computer.

Electrical activity induced in nervous structures by the stimulating pulses is recorded, amplified, and sent to the computer, through an analog-to-digital converter, to submit

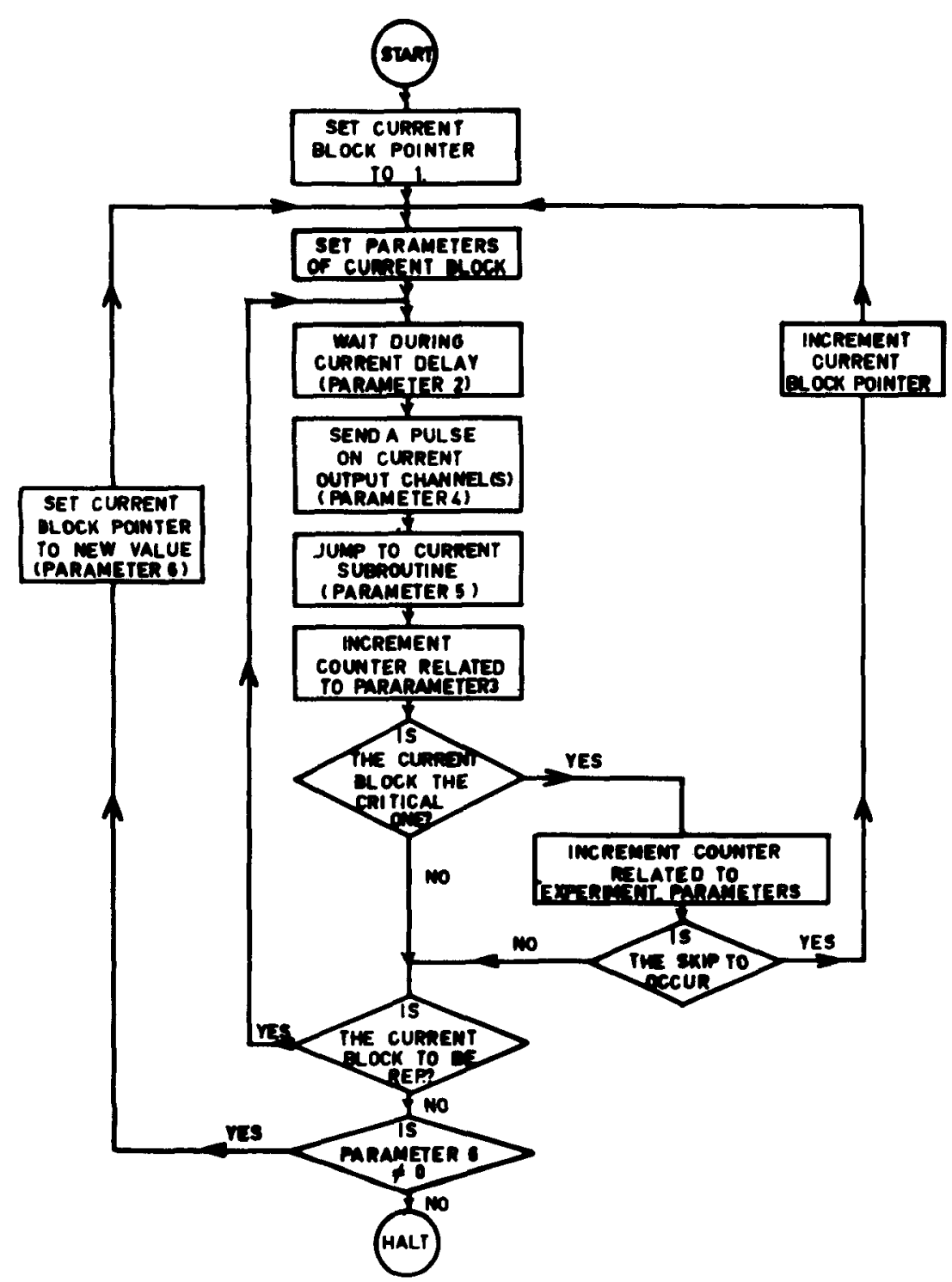

Fig. 1. Flow chart of PPG. them to analysis routines. Thus far, a transient averaging routine and a latency measuring routine have been developed in connection with PPG.

\section{Operation of the PPG}

A flow chart of PPG is shown in Fig. 1. For the sake of programming ease, the PPG has been designed in such a way that each pulse issued on program command by the computer is associated with a routine initiated at the same time. In experimental conditions where this association is not required, either a single pulse may be issued alone by associating it with a no-operation (NOP) routine, or a routine may be started in isolation by sending the corresponding output pulse on a NOP channel.

A pulse-routine association or a repetition of this association at a constant interval constitutes the building block of the PPG, and 


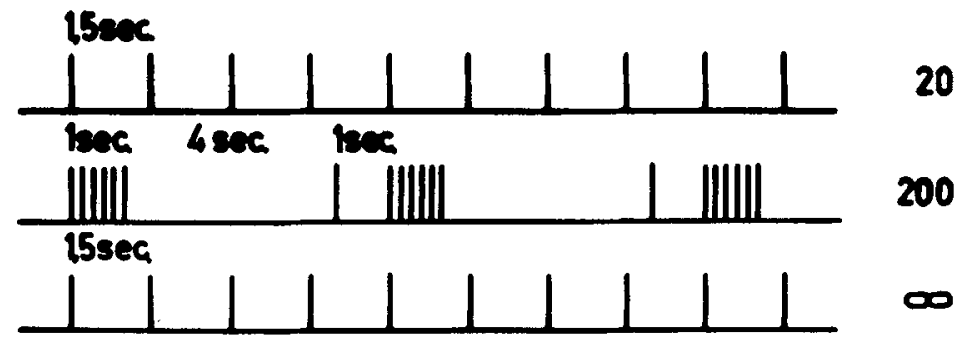

4. 2018
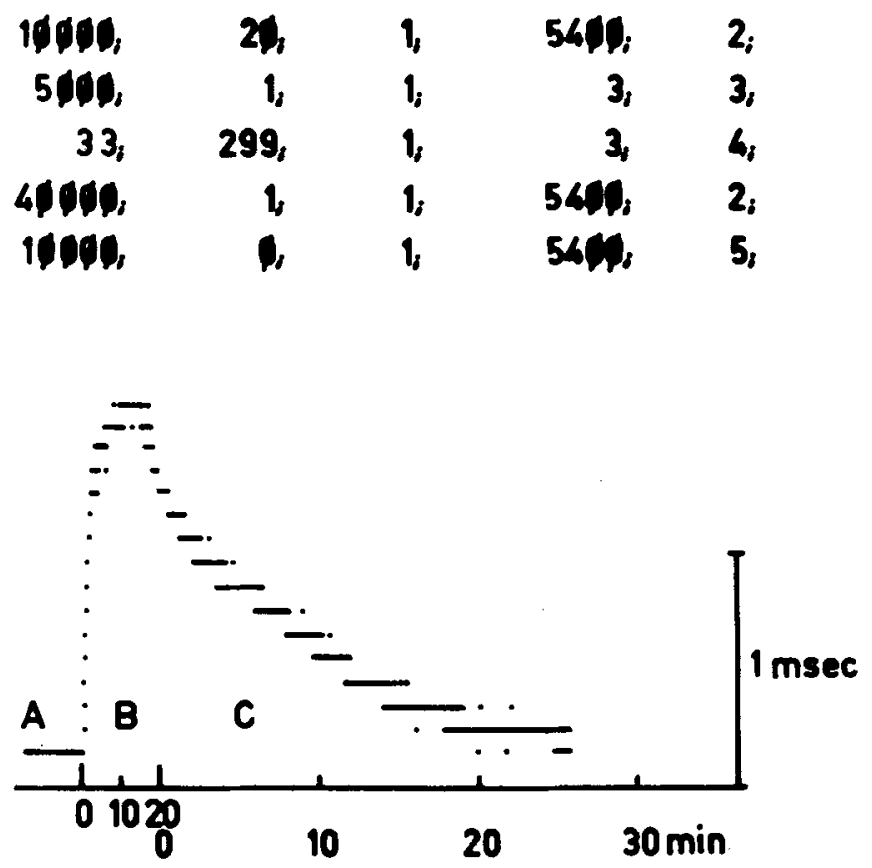

Fig. 2. Lower part of the figure illustrates the changes in latency of a single motor unit of the median nerve activated at the elbow and recorded at the level of the thenar eminance, during the stimulation series illustrated in the upper part of the figure. Middle part of the figure shows the input format for the parameters of this stimulation series. Further explanations are in the text.

programming an experiment merely consists of designing a chronological succession of such building blocks. Each block is defined by six parameters: (1) the block number, to allow reference by other blocks; (2) the interval between each pulse routine association, within the block (when the block is represented by a train of pulses) or the interval after the previous block (when the block is made only of one pulse); (3) the number of repetitions of the pulse-routine association, within the block; (4) the number of the output D/A channel; (5) the start address of the routine started in association with the pulse; and (6) the number of the next block to be considered after execution of the current block.

Programming an experiment consists, then, in dividing it into a series of blocks corresponding to the above definition. In PPG, each of those blocks is considered as a macroinstruction, and jump instructions allow one to plan the execution of the blocks in any desired order, including the possiblity of creating endless loops of blocks. Already within a single block, trains of program to repeat each pulse-routine association as many times as specified with the third parameter of the block. Similarly, the possibility of creating loops of blocks allows one to repeat complex patterns, defined by more than one block. An example of this is shown in the second line of Fig. 2, repeated 200 times, is a train of 300 a single pulse. The number of pulses may be produced by asking the where the elementary pattern, to be pulses at $300 / \mathrm{sec}$, followed at $4 \mathrm{sec}$ by repetitions of the elementary pattern is decided by a conditional skip instruction, which branches the program once the pattern has been repeated a preset number of times.

\section{Input Format of the Parameters}

The parameters of each block of the program are typed sequentially on one line of the Teletype. Above the lines referring to the block parameters, two heading lines refer to the experiment parameters, whose function will be described below. An example of the format is illustrated in Fig. 2.

Block parameters. A given program may contain up to 17 blocks, which are written on consecutive lines of the Teletype and numbered sequentially so that the first parameter of the block must not be typed. The following parameters of the block, as defined above, are typed sequentially: In the first line of the block parameters in Fig. 2, $1 \emptyset . \emptyset \emptyset \emptyset$ represents the interval between pulses, and $2 \emptyset$, the number of repetitions; 1 , the output channel, $54 \emptyset \emptyset$, the start address of the routine, and 2 , the next line to be executed by the program.

The format specifications for the different parameters are as follows: (1) Interval between pulses. The unit of time is 100 microsec; an interval of 2 msec, e.g., is to be coded as $2 \emptyset$. The maximum delay which can be generated by PPG is of the order of $15 \mathrm{~min}$, and the maximum repetition rate is, of course, of $10 \mathrm{KHz}$. When a routine is initiated with the pulse, the interval is measured as the interval between termination of the routine and the delivery of the next pulse. (2) Number of repetitions. This is typed without coding. The maximum value is $10^{7}$. If the operator types $\emptyset$, a quasiinfinite repetition will occur ( 2 to the 24th power). (3) Number of the output channel. Two $A / D$ output channels are used and they are directly numbered as 1 and 2 . Number 3 refers to the possibility of issuing a simultaneous output pulse on 1 and 2 . (4) Start address of the routine. It is indicated in octal value. (5) Number of the next block to be considered by the program. It is typed without coding. When a $\emptyset$ is typed as the 6 th parameter, the program will halt after completion of the current block.

The maximal number of digits which can be accommodated for the different parameters is: 6 for the interval between pulses, 6 for the number of repetitions, 1 for the number of the output channel, 4 for the start address of the routine, and 2 for the number of the next block. The different parameters of a same line are separated by a semicolon sign. The step from one line to the next occurs through a carriage-return/line-feed pair 
(CR-LF). The last line is terminated by a CR-LF followed by a dollar sign, which is not echoed by the computer and causes the program to halt.

As long as the dollar sign is not typed, it is possible to erase the last figures which have been typed by pushing the "question mark" knob as many times as there are figures to be deleted and then continuing with the correct figures.

Experiment parameters. Some parameters related to the entire experiment, rather than to a particular block, are typed in the two heading lines preceding the block parameters. The first line of the heading may contain a $\emptyset$ or a 1 , referring to two different stimulation programs which may be loaded simultaneously in core memory. Only one program is operating at a given time, but fast switching to the other program stored in memory may be achieved by changing manually the position of the 11 th bit of the switch register on the control panel of the PDP-8. The second line of the heading specifies the parameters of the conditional skip instruction mentioned above which is used to issue the program out of a loop of blocks. The first parameter of this line refers to a block number (in the case of Fig. 2, to No. 4, which defines the pulse given $4 \mathrm{sec}$ after the end of the train at $300 / \mathrm{sec}$ ), and the second indicates the number of occurrences required for this block. The interpretation of those parameters may be explained on the basis of the example of Fig. 2: Line 4 is part of a loop between Blocks 2-3-4-2. When Line 4 has been executed 200 times (as specified under Heading Line 2), a conditional skip instruction will lead the program to the next block (Block 5) instead of to Block 2, which is specified at the end of Line 4. This group of experiment parameters allows us, thus, to issue the program out of a loop of blocks, but since a conditional skip instruction can be defined only for one block of the program, it is possible to issue the program out of loop only once. However, this difficulty may be overcome by writing a routine which resets the "experiment parameters" when a first jump out of a loop has occurred. This routine, which is the first to be called after the skip instruction, will replace the stored data corresponding to the second line of the experiment parameters by the data required by the next skip instruction of the program, and which are loaded in known positions of core memory before the start of the experiment.

This indication is given as an example of the versatility of PPG, since it shows that, with the help of short utility routines, it is possible to modify any block or experiment parameter during the course of an experiment. A typical application consists in incrementing progressively an interval parameter in a block for the automatic determination of recovery curves.

\section{Fast Change of the Parameters}

Three possibilities are provided to change rapidly one or several parameters in the program during an experiment. (1) Two stimulation programs may be loaded in core memory, switching from one to the other by operating manually the 11 th switch of the front panel register. (2) Any parameter of the program may be modified during the experiment by including in the blocks routines to modify the parameters when required, as mentioned above. (3) Facilities are provided to modify Parameters 2 (interval) and 3 (number of pulses), but this requires interruption of the experiment. To modify a parameter, the operator has to type sequentially, on one line, the number of the block which contains the parameter to be modified (maximum two digits), a $\emptyset$ or a 1 to specify if the change has to affect Parameter 2 or Parameter 3 , respectively, and finally, the new value of the parameter (maximum six digits). The format is the same as for a normal input, including semicolons, one CR-LF, and one dollar sign. After the typing of those characters, the stimulation program may be restarted from Block 1 .

\section{Organization of the Program}

PPG consists of two main subprograms. The first subprogram takes care of the input of the parameters via the Teletype (keyboard or reader). It transforms the parameters from their easy-to-handle input format into a form usable by the computer and stores them in specific memory locations from which they can be read by the second subprogram when needed.

The second subprogram takes care of the execution of the stimulation and analysis sequence. It operates according to the flow chart of Fig. 1. To follow this flow chart, it is necessary to remember the existence of the "experiment parameters," which allow a conditional skip to occur when a certain "critical" block has run a certain number of times. The routines which are called by reference to Parameter 5 are not a part of PPG, with the exception of a NOP routine. This routine is called to accompany stimulation pulses which are not followed by acquisition or analysis tasks. To be correctly called by PPG, routines other than the NOP have to satisfy two conditions: (1) to be organized as a subroutine to be called by a JMS instruction, and (2) to occupy memory locations left free by PPG.

\section{An Example of Experiment Automatization with PPG}

In the experimental situation chosen as an illustration, the main analysis routine measures the latency of the response of muscle fibers in human hand muscles on stimulating single motor nerve fibers in the median nerve at the elbow (Bergmans, 1970). The aim of the experiment is to study the influence of short trains of pulses repeated every $6 \mathrm{sec}$ on the latency of single responses (Bergmans \& Michaux, 1970). For this experiment, the stimulation series illustrated in the upper part of Fig. 2 was required. Initially, 20 test pulses are given at $1.5-\mathrm{sec}$ intervals to measure the latency under basal conditions (Line 1). The nerve fiber is then subjected to trains of pulses (at $300 / \mathrm{sec}$ for $1 \mathrm{sec}$ ) repeated every $6 \mathrm{sec}$, and the influence of this procedure is tested by measuring the latency to a single pulse given $4 \mathrm{sec}$ after the end of each train (Line 2). After 200 train repetitions, the subsidence of any effect is studied by returning to a series where only testing pulses are given at 1.5 -sec intervals (Line 3).

The middle part of Fig. 2 shows how this stimulation series has been programmed and the program introduced on Teletype. All the pulses are to be issued on the same output channel and this is specified by the third term of each line of block parameters (1). The first 20 control pulses may be grouped in one single block, since they are separated by a same interval. In the first line of the block parameters, $54 \emptyset 0$ represents the start address of the latency measurement routine. This routine measures the latency, stores the result in core memory, and prints it on Teletype. This operation takes $0.5 \mathrm{sec}$, and accordingly the interval between the end of the routine and the next pulse is 10.000 ( $\mathrm{x} 100$ microsec).

After completion of the first block, the program is referred sequentially to Lines 2, 3, and 4, which define, in order, the first pulse of the train, the 299 latter pulses of the train, and the testing pulse given 4 sec after the end of the train. This pattern has to be repeated, and as a consequence the end of Line 4 refers the program to Line 2. The number of repetitions of this pattern is limited to 200 by the second line of the heading. When this number is achieved, a conditional skip instruction refers the program to Line 5, which is looped throughout 
itself to provide for an infinite number of testing pulses.

During such an experiment, the results of consecutive measurements are stored sequentially in core memory. After the end of the experiment, a subroutine takes care of the display of the memory contents, which is shown in the lower part of the figure. Part A shows the latency level in response to the 20 testing pulses (Line 1 in stimulation series). When interpolating trains of pulses every 6 sec (Part B), the latency of the response increases progressively, and, on turning back to testing pulses alone (in C), the latency reverts slowly to control values.
ADVANTAGES OF THE METHOD AND CONCLUSION

The development of PPG was started to provide the experimenter with stimulus sequences which cannot be realized even with complex batteries of stimulators. In this domain the great power of PPG has proven to be fruitful, particularly in suggesting experimental approaches which are not considered with conventional stimulators. Even for simpler experiments, PPG, by reducing the amount of manual operations, shortens the duration of the experiment, and increases the viability of the stimulation series. We hope that it will provide for further developments in the control of the parameters of the stimulating pulses by the computer and in the domain of interaction between experimental results and the stimulation program.

REFERENCES

BERGMANS, J. The physiology of single human nerve fibres. Unpublished thesis, University of Louvain, 1970.

BERGMANS, J.. \& MICHAUX, J. Hyperpolarization evoked in single human nerve fibres by rhythmically repeated tetanizations. Archives of international Physiology, 1970, in press.

\section{NOTE}

1. List of abbreviations: CR-LF-carriage return/line feed: $D / A-$ digital to analog: JMS-jump to subroutine; NOP-no operation: PDG-programmed pulse generator. 